\title{
Combination of FDG PET/CT and Contrast-Enhanced MSCT in Detecting Lymph Node Metastasis of Esophageal Cancer
}

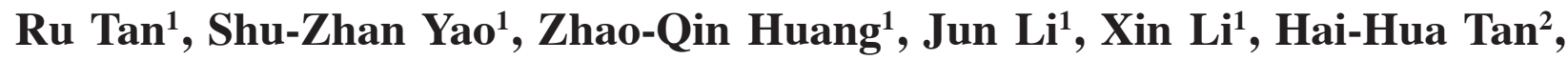 \\ Qing-Wei Liu'*
}

\begin{abstract}
Background: Lymph node metastasis is believed to be a dependent negative prognostic factor of esophageal cancer. To explore detection methods with high sensitivity and accuracy for metastases to regional and distant lymph nodes in the clinic is of great significance. This study focused on clinical application of FDG PET/CT and contrast-enhanced multiple-slice helical computed tomography (MSCT) in lymph node staging of esophageal cancer. Materials and Methods: One hundred and fifteen cases were examined with enhanced 64-slice-MSCT scan, and FDG PET/CT imaging was conducted for neck, chest and upper abdomen within one week. The primary lesion, location and numbers of metastatic lymph nodes were observed. Surgery was performed within one week after FDG PET/CT detection. All resected lesions were confirmed histopathologically as the gold standard. Comparative analysis of the sensitivity, specificity, and accuracy based on FDG PET/CT and MSCT was conducted. Results: There were 946 lymph node groups resected during surgery from 115 patients, and 221 were confirmed to have metastasis pathologically. The sensitivity, specificity, accuracy of FDG PET/CT in detecting lymph node metastasis were $74.7 \%, 97.2 \%$ and $92.0 \%$, while with MSCT they were $64.7 \%, 96.4 \%$, and $\mathbf{8 9 . 0} \%$, respectively. A significance difference was observed in sensitivity $(p=0.030)$, but not the others $(p>0.05)$. The accuracy of FDG PET/CT in detecting regional lymph node with or without metastasis were $91.9 \%$, as compared to $89.4 \%$ for MSCT, while FDG PET/CT and MSCT values for detecting distant lymph node with or without metastasis were $94.4 \%$ and $94.7 \%$. No significant difference was observed for either regional or distant lymph node metastasis. Additionally, for detecting para-esophageal lymph nodes metastasis, the sensitivity of FDG PET/CT was $72 \%$, compared with $54.7 \%$ for MSCT $(p=0.029)$. Conclusions: FDG PET/CT is more sensitive than MSCT in detecting lymph node metastasis, especially for para-esophageal lymph nodes in esophageal cancer cases, although no significant difference was observed between FDG PET/CT and MSCT in detecting both regional and distant lymph node metastasis. However, enhanced MSCT was found to be of great value in distinguishing false negative metastatic lymph nodes from FDG PET/CT. The combination of FDG PET/CT with MSCT should improve the accuracy in lymph node metastasis staging of esophageal cancer.
\end{abstract}

Keywords: Esophageal cancer-lymph node metastasis - MSCT - FDG PET/CT - combination

Asian Pac J Cancer Prev, 15 (18), 7719-7724

\section{Introduction}

Esophageal cancer is the ninth malignant tumor in death in the world. There is an average of 150,000 people died of esophageal cancer every year and ranked forth in China. The 5-year survival rate is less than $10 \%$ for patients suffering from esophageal cancer, and showed poor prognosis. The reason may be due to that the majority of patients have been diagnosed as advanced when perceived, and full-thickness esophageal wall has been involved or lymph node metastasis and distant metastasis has occurred. Lymph node metastasis and metastatic numbers are believed as dependent negative prognostic factors for esophageal cancers (Jiang et al., 2012). Literatures showed that the 5-year survival rate of esophageal cancers with metastatic lymph nodes was less than $15 \%$, compared with that of more than $40 \%$ in patients without (Saha et al., 2001; Feng et al., 2013; Tanaka et al., 2013; Aranda et al., 2014). Therefore, improving the accuracy of lymph node staging in patients with esophageal cancers is of great significance.

Lymph node biopsy based on thoracoscopy and imaging methods are applied widely in clinic for lymph node staging for esophageal cancer. Thoracoscopic lymph node biopsy showed high sensitivity and specificity for obtaining lymph nodes directly and conducting 
pathological examination. However, for the lower esophageal, laparoscopic combination is usually needed additionally, and other inspection methods is essential for the evaluation of the neck and supra-clavicular lymph node metastasis. This takes a long time, and has not yet been widely accepted for invasiveness.

Imaging methods are the most important non-invasive diagnosis for esophageal cancer and lymph node staging. CT and EUS (endoscopic ultrasonography) are currently used commonly as preclinical evaluation imaging methods. CT is used most widely in the staging of esophageal cancer, it can show the size, number and anatomical location of the enlarged lymph nodes clearly, and determine the staging of lymph node combined with morphological changes. However, their effect is not very satisfactory, because enlarged lymph nodes may not be caused by cancer metastasis, and tumors could be existed in normal-sized lymph nodes (Choi et al., 2010). Additionally, enhanced scan is usually needed to distinguish metastatic lymph nodes and normal structures, but patients allergic to iodine can not perform this examination. Some advantages could also be exhibited in EUS detection, such as the accuracy of the depth of involvement of the esophageal wall, and the high sensitivity in the judgment of lymph node metastasis. However, the accuracy is relatively lower, and the effect is usually influenced by instrument performance, operator technique and diagnostic criteria. If esophageal stenosis, the probe usually can not achieve, and therefore evaluation often failed (Yen et al., 2012). Therefore, to explore further imaging methods is of great importance for accurate assessment of esophageal disease, and improving the 5-year survival rate.

The main challenge of imaging methods is failing to determine whether or not cancer cells existed in the lymph nodes whose shortest diameter smaller than the diagnostic criteria, and metastatic or inflammatory response in the lymph nodes whose shortest diameter greater than the diagnostic criteria. As a functional imaging, FDG PET (positron emission tomography using [18F] fluorodeoxyglucose, FDG PET) can reflect metabolic changes in tissue sensitively, and showed higher sensitivity in diagnosis for the earlier occurrence of metabolic than the morphological changes in tissue lesions (You et al., 2013). Yasuda. et al. reported the first application of FDG PET in esophageal cancer lymph node staging (Yasuda et al., 1995), and higher accuracy was proved by more studies compared with CT and EUS (Kato et al., 2002). However, PET imaging is still a low data volume, low-resolution, lownoise ratio method, the image is grainy, and anatomical structure shows poor. Diagnostician often can not focus accurately, especially in the junctions, based on PET imaging results. PET and CT image fusion method was used for further positioning. But the two images were collected at different time, the poor time and space coordination is inevitable. Additionally, some lesions seen in PET may be normal in the CT, brought difficulty to diagnosis based on the visual integration.

Later, PET and CT was installed on the same instrument, which named as PET/CT. PET images which reflect the metabolic function of tissue and CT images to display anatomical detail were collected under the same scan respectively. PET/CT fused image was obtained, achieved the matching of anatomical and functional details, and may reflect both the biological and morphological changes in early detection of disease. PET/ CT has been used widely in the diagnosis and clinical staging of the tumors in clinic.

In this study, we assessed the combination value of FDG PET/CT and contrast-enhanced MSCT in detecting regional and distant lymph node metastasis from 115 cases of esophageal cancers. FDG PET/CT showed more sensitive than MSCT in detecting lymph node metastasis, especially for para-esophageal lymph nodes in esophageal cancer, although no significant difference was observed between FDG PET/CT and MSCT in detecting both regional and distant lymph node metastasis. But enhanced MSCT was found to be of great value in discovering false negative metastatic lymph nodes from FDG PET/CT. So, we proposed the combination of FDG PET/CT and MSCT to improve the accuracy in lymph node metastasis status of esophageal cancer.

\section{Materials and Methods}

\section{General information}

A total of 115 patients with thoracic esophageal cancer (71 male and 44 female cases) from the Shandong provincial hospital affiliated with Shandong University in the period of 2005.9-2013.6 were studied. There were 20 cases of upper thoracic, 72 cases of thoracic, and 23 cases of lower thoracic esophageal cancers. The mean age of the patients was 57.9 (range 45-76). All of these patients have not been suffered from any other malignant tumors in 5 years. Enhanced MSCT was performed first, and 18F-FDG PET/CT was conducted into one week. Surgical resection was operated into one week after 18F-FDG PET/ CT assay. All tissue specimens were embedded in paraffin for pathological examination.

\section{Examination methods}

18F-FDG PET/CT scans were obtained on a PET/CT scanner (GE Discovery LS4 PET/CT, General Electrical Medical Systems). After at least 6 hour fasting, 10-15 $\mathrm{mCi} 18 \mathrm{~F}-\mathrm{FDG}$ (radiochemical purity of $>98 \%$ ) was administered (5.55 MBq/kg). Data acquisition started 40 min after injection in whole body mode (i.e. from the neck to the pelvis). Before examination, $10 \mathrm{mg}$ Anisodamine was administered intramuscularly preceding 20 minutes to emission scanning, and patients were asked to drink $500 \mathrm{ml}$ water to make stomach full. First 4 slices spiral CT was performed, $140 \mathrm{Kv}, 100 \mathrm{~mA}$, pitch=5.Then PET images were collected in two-dimensional mode after CT attenuation correction for 4-minute per bed. All the data were transferred to the workstation (GE Xeleris). After iterative reconstruction, images of PET, CT and fused PET/CT were obtained in trans-axial, coronal and sagittal views.

Conventional CT images were acquired with a 64-detector-row CT scanner (VCT light-speed 64; GE Healthcare, USA) mean one week before PET/CT. The following parameters were applied: collimation $\mathrm{v} \mathrm{mm}$, rotation time $0.35 \mathrm{~s}$, tube voltage $120 \mathrm{kV}$, tube current 
$300 \mathrm{~mA}$, and pitch 1.375. Intravenous contrast agent (Optiray, $320 \mathrm{mgI} / \mathrm{mL}$ ) was injected into an antecubital vein at a flow rate of $2.5 \mathrm{ml} / \mathrm{s} .5-\mathrm{mm}$ contiguous images were obtained from the throat to the level of umbilicus. The delayed time was 50s. All images were transferred to the workstation (Advantage Windows 4.4, GE Medical Systems), and the reconstruction programs MPR (MultiPlanar Reformatting)was used with reconstruction thickness $1.25 \mathrm{~mm}$ and $1 \mathrm{~mm}$ overlap.

\section{Image analysis}

Image validation was performed blindly and without knowledge of the eventual clinical parameters by three PET/CT physicians independently. When differences between inter-observers occurred, the images in question were jointly re-examined by three investigators. Images were read from computer monitor directly to observe the abnormal uptake of radioactive FDG in esophagus, compared with that in adjacent muscle tissues. The result was divided into 3 levels: A. none: no radioactive uptake; B. questionable: with comparable level in muscle; C. definite: higher uptake than muscle, seen to be positive samples. Locations of positive image, the depth of primary tumor invasion, and adjacent tissue status were recorded. Other local gathered lesions from the PET/CT images were investigated, to compare their radioactive uptake levels with that in mediastinum. Positive sample means the higher uptake level than mediastinum, and negative in converse. Positive lymph nodes were decided and located accurately in the fusing PET/CT image. Grouping of lymph node was based on Korst's reduced grouping method. Abnormal imaging lesions were observed at the same time. For quantitative evaluation, the highest level of cross-section of all positive lesions were selected, taken $90 \%$ area of the lesion as the region of interest (ROI). Standard uptake value (SUV) was calculated automatically by computer. Malignances were taken into consideration if $\mathrm{SUV}>2.5$, but comprehensive assessment should be necessary, including the distribution consistence and edge of radionuclide. Malignant lesion was definite only when the radionuclide distributed uniformly and edge clearly under the precondition of $\mathrm{SUV}>2.5$.

MSCT images were reviewed by 3 experienced radiologists without the knowledge of clinical information. The criteria of positive lymph nodes diagnosis was as follows: (1) short diameter $>10 \mathrm{~mm}$; (2) any sulcus oesophageal lymph node; (3) enhanced lymph node with thin wall and ring shaped. Benignant lymph nodes were taken into consideration when fat density exhibited in the center or when exhibited as heterogeneous enhancement with thick edge after enhancement. The grouping criteria of lymph nodes was consistent with that of PET/CT.

\section{Statistical analysis}

Pathological examination was gold standard. The sensitivity, specificity, and accuracy of PET/CT and MSCT for detecting lymph node metastasis were calculated by comparing the results of each scan with results of FNAB or pathologic examination of each resection specimen. Moreover the sensitivities of the two methods was calculated. $\mathrm{X}^{2}$ test was performed under SPSS 16.0 software. $\mathrm{P}$ values less than 0.05 were considered significant.

\section{Results}

\section{Pathological examinations}

Among the 115 cases of esophageal cancer, pathological types were distributed as follows: 108 cases of squamous cell carcinoma, 4 cases of adenocarcinoma, and 3 cases of adeno-squamous carcinoma. After surgery, 946 groups of lymph nodes were removed (2923 cases, short diameter 4 $\sim 22 \mathrm{~mm}$ ), and 221 groups (623 cases, average diameter $11 \mathrm{~mm}$ ) showed tumor metastasis pathologically.

\section{Diagnostic value of PET/CT and MSCT for lymph node metastasis}

One hundred and eighty-five groups of lymph nodes were identified positive by PET/CT, with 165 groups actually positive. One hundred and sixty-nine groups lymph nodes were identified positive by enhanced MSCT, and the true positive group number was 143 . Comparisons between FDG PET/CT or MSCT detections and pathological diagnosis in 946 groups of lymph nodes were exhibited as Table 1 . The diagnostic sensitivity and specificity of PET/CT were $74.7 \%(165 / 221)$ and $97.2 \%$ (705/725), with that of MSCT as $64.7 \%(143 / 221)$ and $96.4 \%(699 / 725)$. Significance in statistics was only obtained in the sensitivity of the two methods $(p=0.030)$.

Depending on the scope of metastasis, lymph nodes were classified into regional lymph nodes, which was

Table 2. Comparisons between FDG PET/CT and MSCT in Regional (N) and Distant (M) Lymph Nodes Detections with or without Metastasis

\begin{tabular}{|c|c|c|c|c|c|c|c|c|}
\hline Detection & \multicolumn{2}{|c|}{$\mathrm{N}+$} & \multicolumn{2}{|c|}{ M1 } & \multicolumn{2}{|c|}{ No } & \multicolumn{2}{|c|}{ M0 } \\
\hline methods & $\mathrm{TP}$ & FN & TP & FN & $\mathrm{TN}$ & FP & $\overline{\mathrm{TN}}$ & $\overline{\mathrm{FP}}$ \\
\hline $\mathrm{PET} / \mathrm{CT}$ & 141 & 46 & 21 & 13 & 450 & 6 & 265 & 4 \\
\hline MSCT & 120 & 67 & 20 & 14 & 455 & 1 & 267 & 2 \\
\hline Total & \multicolumn{4}{|c|}{221} & \multicolumn{4}{|c|}{725} \\
\hline
\end{tabular}

*TP: True Positive; FN: False Negative; TN: True Negative; FP: False Positive $\mathrm{N}+$ : metastic regional lymph nodes; $\mathrm{NO}$ : regional lymph nodes without metastasis; M1: metastic distant lymph nodes; M0: distant lymph nodes without metastasis.

Table 1. General Information about FDG PET/CT and MSCT with Pathological Diagnosis in Lymph Nodes Metastasis of Esophageal Cancers

\begin{tabular}{|c|c|c|c|c|c|c|c|}
\hline \multicolumn{2}{|c|}{ Detection methods } & \multicolumn{2}{|c|}{ pathological examination } & \multirow[t]{2}{*}{ Total } & \multirow[t]{2}{*}{ sensitivity } & \multirow[t]{2}{*}{ specificity } & \multirow[t]{2}{*}{ accuracy } \\
\hline & & + & - & & & & \\
\hline \multirow{2}{*}{$\mathrm{PET} / \mathrm{CT}$} & + & 165 & 20 & 185 & $74.7 \%$ & $97.2 \%$ & $92.0 \%$ \\
\hline & - & 56 & 705 & 761 & & & \\
\hline \multirow[t]{2}{*}{ MSCT } & + & 143 & 26 & 169 & $64.7 \%$ & $96.4 \%$ & $89.0 \%$ \\
\hline & - & 78 & 699 & 777 & & & \\
\hline$P$ value & & & & & 0.030 & $>0.05$ & $>0.05$ \\
\hline
\end{tabular}




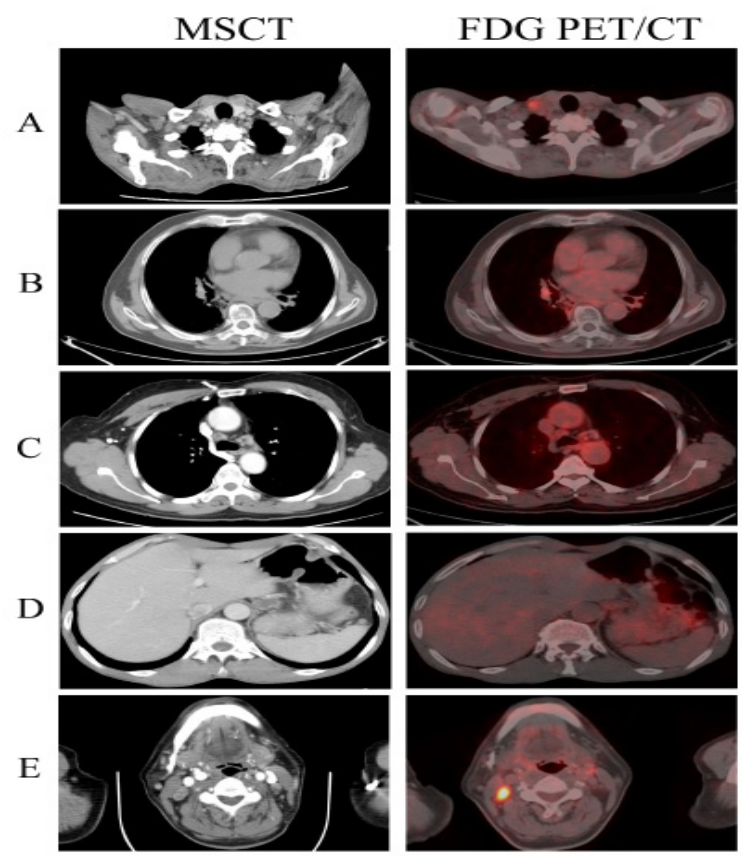

Figure 1. Some Representative Visions for FDG PET/ CT and MSCT in Detecting Lymph Nodes Metastasis. A) The metastatic lymph nodes could be found in the right subclavian of a lower thoracic esophageal squamous cell carcinoma patient. MSCT showed the lymph node with ring shaped enhancement, the short diameter of which was about $13 \mathrm{~mm}$, and high uptake could be found under FDG PET/CT; B) A false positive imaging in right hilum of a lower thoracic esophageal squamous cell carcinoma patient was showed. MSCT scanned a high-density lymph node in right hilum, with the short diameter about $5 \mathrm{~mm}$, but high radioactive uptake could be found under FDG PET/CT. Pathological diagnosis proved no metastasis; C) A benign enlarged mediastinal lymph node field was showed in one middle thoracic esophageal squamous cell carcinoma patient. MSCT showed many lymph nodes located in behind of the superior vena cava and in primary pulmonary window. A ring shaped enhanced lymph node behind of superior vena cava was scanned, the short diameter of which was about $12 \mathrm{~mm}$. No radioactive uptake was obtained by FDG PET/CT; D) A benign enlarged mediastinal lymph node field was showed in one middle thoracic esophageal squamous cell carcinoma patient. MSCT showed many lymph nodes located behind of the superior vena cava and in primary pulmonary window. A ring shaped enhanced lymph node behind of superior vena cava was scanned, the short diameter of which was about $12 \mathrm{~mm}$. No radioactive uptake was obtained by FDG PET/CT; E) Bilateral cervical lymph node metastasis could be seen in a upper thoracic esophageal squamous cell carcinoma patient. MSCT scanned a ring shaped enhanced lymph node with the short diameter about $11 \mathrm{~mm}$ in the right carotid artery sheath, and a uniform enhanced lymph node with the short diameter about $7 \mathrm{~mm}$ in the left carotid artery sheath. Both of these two lymph nodes showed radioactive uptake under FDG PET/CT

Table 3. Comparisons between FDG PET/CT and MSCT in Para-Esophageal Lymph Nodes Detections $(\mathbf{p}=\mathbf{0 . 0 2 9})$

\begin{tabular}{lrrr}
\hline FDG PET/CT & \multicolumn{2}{c}{ MSCT } & Total \\
& + & - & \\
\hline+ & 35 & 19 & 54 \\
- & 6 & 15 & 21 \\
Total & 41 & 34 & 75 \\
\hline
\end{tabular}

classified as $\mathrm{N}$ staging, and distant lymph nodes, which was involved in distant metastasis (M). Here, comparisons between FDG PET/CT and MSCT detections in regional and distant lymph nodes with or without metastasis were also analyzed in Table 2. The accuracy of FDG PET/CT in detecting regional lymph node with or without metastasis were $91.9 \%(591 / 643)$, which was $89.4 \%(575 / 643)$ in MSCT. While the accuracy of FDG PET/CT and MSCT in detecting distant lymph node with or without metastasis were $94.4 \%$ (286/303) and 94.7\% (287/303), respectively. No significant difference was observed in both regional and distant lymph node metastasis under FDG PET/CT and MSCT detections $(p>0.05)$.

Some representative visions were also exhibited in Figure 1, including both FDG PET/CT and MSCT true positive imaging (Figure 1-A), FDG PET/CT (Figure 1-B) or MSCT (Figure 1-C) false positive imagings in detecting lymph nodes without metastasis, FDG PET/CT (Figure

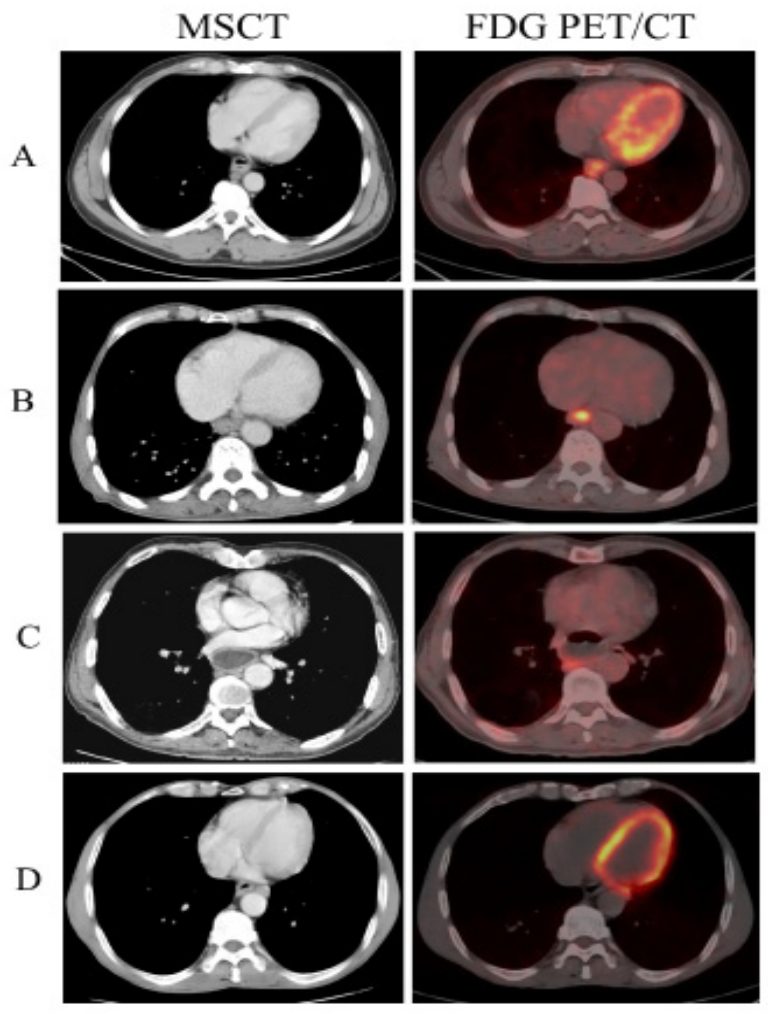

Figure 2. Some Representative Visions for FDG PET/ CT and MSCT in Detecting Para-Esophageal Lymph

Nodes Metastasis. A) A lower thoracic esophageal squamous cell carcinoma patient with metastatic para-esophageal lymph nodes. MSCT showed a lymph node with ring shaped enhancement behind of the esophagus, the short diameter of which was about $9 \mathrm{~mm}$. Lesions in the esophagus and high uptake could be found under FDG PET/CT. B) A lower thoracic esophageal squamous cell carcinoma patient with metastatic para-esophageal lymph nodes was exhibited. MSCT showed a lymph node with the short diameter about $7 \mathrm{~mm}$ in the front of the esophagus. FDG PET/CT proved this high uptake as lesions in the esophagus, without imaging. C) A lower thoracic esophageal squamous cell carcinoma patient with metastatic para-esophageal lymph nodes was showed. MSCT showed a lymph node with the short diameter about 6 $\mathrm{mm}$ in the behind of the esophagus, but high radioactive uptake could be found under FDG PET/CT.D) A middle thoracic esophageal squamous cell carcinoma with metastatic para-esophageal lymph nodes. MSCT showed a lymph node with the short diameter about $12 \mathrm{~mm}$ in paraesophagus, located between the pericardium and the thoracic aorta. But no imaging was found under FDG PET/CT. 
1-D) or MSCT (Figure 1-E) false negative imagings in detecting metastatic lymph nodes.

\section{Diagnostic value of PET/CT and MSCT for lymph node metastasis in para-esophageal regions}

There were 145 groups of lymph nodes located in para-esophageal areas totally (short diameter $4 \sim 12 \mathrm{~mm}$, averaged $8 \mathrm{~mm}$ ), with 75 groups metastasis. As shown in Table 3, PET/CT and MSCT diagnosed 54 and 41 groups accurately, respectively. The sensitivity of PET/CT was $72.0 \%(54 / 75)$ and that of MSCT was $54.7 \%$ (41/75) in the diagnosis of para-esophageal lymphs, with significant difference $(p=0.029)$.

Among the 54 positive groups of PET/CT, there were 35 groups consistent with MSCT (Figure 2-A), and 19 groups which can not be distinguished from the primary tumor on MSCT images alone (Figure 2-C). Among the 41 groups of MSCT, there were 15 negative groups, consistent with PET/CT (Figure 2-B), and 6 groups which can not be distinguished from the primary tumor on PET/ CT images alone

\section{Discussion}

CT is the most widely used method in diagnosing the preoperative staging of esophageal cancer. MSCT has the advantage of isotropic which makes it possible to show the node morphous in different aspect by MPR, to show and measure the short diameters accurately, and to combine the criteria of short diameter with the enhanced future for the accurate diagnosis. Although the criteria is different as the different investigator, the common method to detect whether or not metastasis existed is to measure its short diameter. Mizowaki. et al. compared the enlargement of lymph node based on CT scanning with that diagnosed by pathological examination in 41 cases of esophageal cancers, proposing $5 \mathrm{~mm}$ as the diagnostic criteria of short diameter, the sensitivity and specificity of which were $68 \%$ and $87 \%$, respectively (Mizowaki et al., 1996). Currently most investigators take $10 \mathrm{~mm}$ as the lower limit of lymph node metastasis, with the sensitivity and specificity of which are 11\% 77\% and 71\% 97.3\%, respectively (Yoon et al., 2003; Okada et al., 2009; Ma et al., 2013). The enhanced features of metastatic lymph nodes from different pathology of primary tumors showed different under MSCT scanning, usually displaying as ring-shaped and thin-wall enhancement. Meanwhile, lymph nodes from tuberculosis necrotizing lymphadenitis show heterogeneous enhancement with thick edge (Lee et al., 2012). So, MSCT is able to diagnose lymph node metastasis. Here we showed consistent diagnostic sensitivity and specificity with previous literatures. In other way, the size of lymph nodes from different position has no uniform standards, and not only tumor metastasis but also infection and reactive hyperplasia could enlarge lymph nodes. Metastasis may existed in nodes the short diameter of which less than diagnostic criteria. All of those brings difficulty to diagnosticians.

Compared with previous literatures (Manabe et al., 2013; Li et al., 2014), both the sensitivity and the specificity of FDG PET/CT are higher in this study. The reason may be related with detecting method and poor imaging based on PET with common CT or monolayer spiral CT. Additionally, the scanning slice thickness of conventional CT is $5 \mathrm{~mm} \sim 10 \mathrm{~mm}$, and the image quality is easy to be affected by partial volume effect. Also, it is difficult to clearly display node morphous and features inside only through trans-axial view.

In this study, 56 groups of lymph nodes exhibited false negative in 18F-FDG PET/CT analysis. The reason may be that: (1) less tumor cells in metastatic lymph node, which leading to the less 18F-FDG uptake; (2) the metastatic lymph node is small in size, which is easily limited by PET spatial and affected by partial volume effect; (3) there is a great area of necrosis in metastatic lymph nodes. This study suggests that combining 18F-FDG PET/CT with MSCT may evaluate the accuracy of $\mathrm{N}$ staging.

There are also some false positive cases in both 18F-FDG PET/CT and MSCT analysis in this study. The false positive rate is $6.34 \%(46 / 725)$, consistent with previous reports (Lerut et al., 2000; Kim et al., 2001). The reason may be that: $18 \mathrm{~F}-\mathrm{FDG}$ is not specific in labeling tumor cells, enlarged lymph nodes due to some unspecific infections could also take up 18F-FDG excessively, exhibited with positive imaging. Especially for elder patients, the lymph nodes which lies in the hilum of lung often is diagnosed as chronic reactive lymphadenopathy pathologically. The main points of differential diagnosis are that reactive lymphadenopathy node usually located in hilum of lung, and it has uniformly high density because of calcium deposition in MSCT.

Based on the 7th edition of esophageal cancer staging published in 2009 from American Joint Committee on Cancer (AJCC) and Union for International Cancer Control (UICC), lymph nodes in esophageal cancer were devided into regional lymph nodes $(\mathrm{N})$, which was staged as N0, N1, N2, and N3 depending on the number of metastic lymph nodes, and distant lymph nodes, which was involved in distant metastasis (M) and was classified as M1 and M0 depending on metastasis or not. Here we investigated the accuracy of regional metastic lymph nodes $(\mathrm{N}+)$ and lymph nodes without metastasis (N0) under FDG PET/CT and MSCT detections, and no significant difference was observed. At the same time, we studied the FDG PET/CT and MSCT function in detecting distant lymph nodes metastasis, including M0 and M1. It is noted that M1 stage in this studied only mean metastasis diagnozed in distant lymph nodes, not including the other distant metastasis. Results showed no significance between FDG PET/CT and MSCT in detecting distant metastasis of esophageal cancer. Our results were supported by Schreurs' study, which showed that the number of regional and distant lymph node metastasis detected by PET/CT did not have an influence on lymph node staging of esophageal cancer (Schreurs et al., 2008). At the same time, our findings seems to be inconsistent with previous reports published in Chinese Journal of Medical Imaging by professor Huang, which declared the significant differences between FDG PET/CT and CT in detecting the staging of esophageal cancer. However, CT scanning we conducted here was contrast-enhanced MSCT, which was believed to be more sensitive and 


\section{Ru Tan et al}

specific than tradditional CT scanning. So, it is possible for contrast-enhanced MSCT to be more accurate than tradditional CT in detecting lymph node metastasis. This may be the main reason for this inconsistence.

Documents have reported that false negative node in para-esophagus has a high proportion in 18F-FDG PET imaging (Luketich et al., 1997). The reason was that metastatic lymph node is too close with primary foci, so that the higher uptake of primary foci covered up that of metastatic lymph nodes, exhibited with false negative imaging. PET/CT combines the higher sensitivity of PET with the higher resolution of CT. Therefore partial lymph nodes which cannot be discriminate from primary tumor may be displayed positively under PET/CT. This suggests that PET/CT has higher sensitivity than PET in diagnosis of para-esophageal lymph node metastasis.

Above all, the combination of 18F-FDG PET/CT with MSCT to evaluate the lymph node metastasis of esophageal cancer is not only benefit for preoperative staging but also be helpful for studies in other regions of lymph nodes study.

\section{Acknowledgements}

This study was approved by Shandong Province Science and Technology Development Program (No. 2012GSF11820, No.2012YD18053, No. 2012YD18086 ).

\section{References}

Aranda-Hernandez J, Cirocco M, Marcon N (2014). Treatment of dysplasia in barrett esophagus. Clin Endosc, 47, 55-64.

Choi J, Kim SG, Kim JS, Jung HC, Song IS (2010). Comparison of endoscopic ultrasonograph (EUS), positron emission tomography (PET), and computed tomography (CT) in the preoperative locoregional staging of resectable esophageal cancer. Surg Endosc, 24, 1380-86.

Feng JF, Huang Y, Chen QX (2013). Prognostic value of preoperative serum CA 242 in esophageal squamous cell carcinoma cases. Asian Pac J Cancer Prev, 14, 1803-6.

Jiang JT, Zhang LF, Zhou B, et al (2012). Relationships of uPA and VEGF expression in esophageal cancer and microvascular density with tumorous invasion and metastasis. Asian Pac J Cancer Prev, 13, 3379-83.

Kato H, Kuwano H, Nakajima M, et al (2002). Comparison between positron emission tomography and computed tomography in the use of the assessment of esophageal carcinoma. Cancer, 94, 921-8.

Kim K, Park SJ, Kim BT, et al (2001). Evaluation of lymph node metastases in squamous cell carcinoma of the esophagus with positron emission tomography. Ann Thorac Sug, 71, 290-4.

Lee S, Yoo JH, Lee SW (2012). Kikuchi disease: differentiation from tuberculous lymphadenitis based on patterns of nodal necrosis on CT. AJNR Am J Neuroradiol, 33, 135-40.

Lerut T, Flamen P, Ectors N, et al (2000). Histopathologic validation of lymph node staging with FDG PET scan in cancer of the esophagus and gastroesophageal junction: A prospective study based on primary surgery with extensive lymphadenectomy. Ann Surg, 232, 743-52.

Li YM, Lin Q, Zhao L, et al (2014). Pre-treatment metabolic tumor volume and total lesion glycolysis are useful prognostic factors for esophageal squamous cell cancer patients. Asian Pac J Cancer Prev, 15, 1369-73.

Luketich JD, Schauer PR, Meltzer CC, et al (1997). Role of positron emission tomography in staging esophageal cancer. Ann Thorac Surg, 64, 765-9.

Ma JB, Chen EC, Song YP, et al (2013). Prognostic significance of 18F-fluorodeoxyglucose positron emission tomography (PET)-based parameters in neoadjuvant chemoradiation treatment of esophageal carcinoma. Asian Pac J Cancer Prev, 14, 2477-81.

Manabe O, Hattori N, Hirata K, et al (2013). Diagnostic accuracy of lymph node metastasis depends on metabolic activity of the primary lesion in thoracic squamous esophageal cancer. $J$ Nucl Med, 54, 670-6.

Mizowaki T, Nishimura Y, Shimada Y, et al (1996). Optimal size criteria of malignant lymph nodes in the treatment planning of radiotherapy for esophageal cancer:evaluation by computed tomography and magnetic resonance imaging. Int J Radiat Oncol Biol Phys, 36, 1091-98.

Okada M, Murakami T, Kumano S, et al (2009). Integrated FDG-PET/CT compared with intravenous contrast-enhanced $\mathrm{CT}$ for evaluation of metastatic regional lymph nodes in patients with resectable early stage esophageal cancer. Ann Nucl Med, 23, 73-80.

Saha S, Dehn TC (2001). Ratio of invaded to removed lymph nodes as a prognostic factor in adenocarcinoma of the distal esophagus and esophagogastric junction. Dis Esophagus, 14, 32-36.

Schreurs LM, Pultrum BB, Koopmans KP, et al (2008). Better assessment of nodal metastases by PET/CT fusion compared to side-by-side PET/CT in oesophageal cancer. Anticancer Res, 28, 1867-73.

Tanaka T, Matono S, Mori N, Shirouzu K, Fujita H (2013). T1 squamous cell carcinoma of the esophagus: long-term outcomes and prognostic factors after esophagectomy. Ann Surg Oncol, 21, 932-8.

Yasuda S, Raja S, Hubner KF (1995). Application of whole-body positron emission tomography in the imaging of esophageal cancer: report of a case. Surg Today, 25, 261-4.

Yen TJ, Chung CS, Wu YW, et al (2012). Comparative study between endoscopic ultrasonography and positron emission tomography-computed tomography in staging patients with esophageal cell carcinoma. Dis Esophagus, 25, 40-47.

Yoon YC, Lee KS, Shim YM, et al (2003). Metastasis to regional lymph nodes in patients with esophageal squamous cell carcinoma: CT versus FDG PET for presurgical detection prospective study. Radiology, 227, 764-70.

You JJ, Wong RK, Darling G, et al (2013). Clinical utility of 18F-fluorodeoxyglucose positron emission tomography/ computed tomography in the staging of patients with potentially resectable esophageal cancer. $J$ Thorac Oncol, 8, 1563-69. 